\title{
Biochemical Effects of Levothyroxine Withdrawal in Patients with Differentiated Thyroid Cancer
}

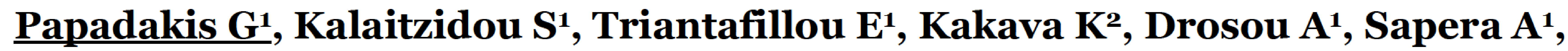

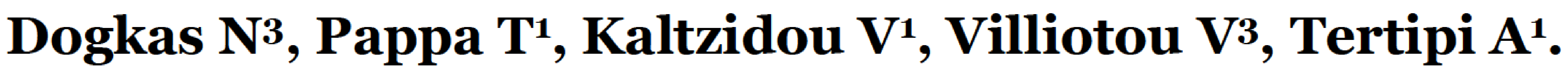 \\ Department of Endocrinology ${ }^{1}$, Otolaryngology ${ }^{2}$ and Biochemistry ${ }^{3}$ \\ Metaxa Anticancer Hospital, Piraeus, Athens, Greece.
}

Table I. Values of the various biochemical parameters off and on levothyroxine (LT4) in the entire study population.

\begin{tabular}{lcccc}
$\begin{array}{l}\text { Parameter } \\
(\mathrm{N}=345)\end{array}$ & $\begin{array}{c}\text { Reference } \\
\text { range }\end{array}$ & $\begin{array}{c}\text { Off LT4 } \\
\text { (mean } \pm \text { SD) }\end{array}$ & $\begin{array}{c}\text { On LT4 } \\
\text { (mean } \pm \text { SD) }\end{array}$ & $p$-Value \\
\hline Glucose (mg/dl) & $70-100$ & $94.7 \pm 25.2$ & $101.6 \pm 29.1$ & $<0.001$ \\
Creatinine (mg/dl) & $0.6-1.1$ & $0.97 \pm 0.23$ & $0.75 \pm 0.15$ & $<0.001$ \\
Uric acid (mg/dl) & $2.6-6.0$ & $6.0 \pm 1.9$ & $5.3 \pm 2.9$ & $<0.001$ \\
t-Cholesterol (mg/dl) & $<200$ & $316.1 \pm 73.5$ & $203.5 \pm 41.2$ & $<0.001$ \\
TGL (mg/dl) & $<150$ & $201.1 \pm 155.4$ & $116.9 \pm 56.2$ & $<0.001$ \\
HDL-c (mg/dl) & $>40$ & $66.6 \pm 16.2$ & $50.6 \pm 12.4$ & $<0.001$ \\
LDL-c (mg/dl) & $<130$ & $208.9 \pm 62.7$ & $129.5 \pm 36.5$ & $<0.001$ \\
VLDL-c (mg/dl) & $<32$ & $41.6 \pm 32.5$ & $24.0 \pm 14.3$ & $<0.001$ \\
AST (U/l) & $10-40$ & $33.8 \pm 16.2$ & $19.1 \pm 8.2$ & $<0.001$ \\
ALT (U/) & $10-35$ & $36.8 \pm 29.6$ & $22.8 \pm 15.6$ & $<0.001$ \\
$\gamma$-GT (U/l) & $<30$ & $33.5 \pm 60.1$ & $28.0 \pm 48.5$ & $<0.001$ \\
LDH (U/l) & $125-245$ & $231.0 \pm 51.1$ & $189.0 \pm 42.6$ & $<0.001$ \\
CPK (U/l) & $38-174$ & $399.9 \pm 697.6$ & $91.6 \pm 68.7$ & $<0.001$ \\
K (mmol/l) & $3.5-5.1$ & $4.19 \pm 0.38$ & $4.27 \pm 0.38$ & 0.001 \\
Na (mmol/l) & $136-145$ & $138.6 \pm 2.2$ & $140.0 \pm 3.6$ & $<0.001$ \\
Albumin (g/dl) & $3.5-5.5$ & $4.35 \pm 0.33$ & $4.24 \pm 0.34$ & $<0.001$ \\
\hline & & & &
\end{tabular}

t-Cholesterol, total cholesterol; TGL, triglycerides; HDL-c, high density lipoprotein cholesterol; LDL-c, low density lipoprotein chlolesterol, VLDL-c, very low density lipoprotein cholesterol; AST, aspartate aminotransferase; ALT, alanine aminotransferase; LDH, lactate dehydrogenase; $\mathrm{CPK}$, creatine phosphate kinase; $\mathrm{K}$, potassium; $\mathrm{Na}$, sodium.
BACKGROUND: Many patients with differentiated thyroid cancer (DTC) are treated with radioiodine (I-131) after thyroidectomy, while they are hypothyroid and all are submitted to withdrawal of LT4 periodically for the evaluation of their disease. Among the tests used for followup is serum thyroglobulin ( $\mathrm{Tg}$ ) as a tumor marker, and occasionally, total body scan with I-131. Maximal sensitivity of the aforementioned tests is established in the hypothyroid state, under elevated thyrotropin (TSH) concentrations, achieved with LT4 withdrawal. The aim of our study was to determine the degree of change in several biochemical parameters due to a short-term but acutely supervening hypothyroidism.

PATIENTS AND METHODS: A total of 345 patients, 60 males (17\%) and 285 (83\%) females, with a history of DTC were enrolled in the study. Their mean age ( \pm SD) was $54.7 \pm 13.6$ years (range $=17-83$ years). Their biochemical profile and serum free triiodothyronine (FT3), free thyroxine (FT4) and thyrotropin (TSH) levels were measured during withdrawal of LT4 treatment, and several months after restarting LT4.

RESULTS: During withdrawal, the intra-individual percentage increase in total cholesterol, low density lipoprotein-cholesterol, very low density lipoproteincholesterol and triglycerides was of the order of $60-80 \%$ and that for high density lipoprotein-cholesterol $30 \%$. Creatinine increased by $30 \%$, whereas $\mathrm{Na}$ and $\mathrm{K}$ levels decreased by $1 \%$. The increase for creatinine phosphate kinase was around 200-300\%, for aspartate aminotransferase and alanine aminotransferase 50-80\%, for $\gamma$-glutamyl transpeptidase $10-20 \%$, and for lactate dehydrogenase 25\%. Glucose decreased by $1-4 \%$. Table 1 shows the values of various biochemical parameteres off and on LT4.

CONCLUSION: Acute, short-term hypothyroidism has significant impact on many biochemical parameters, reflecting the relative alterations in many organ functions and metabolic pathways. Patients with borderline biochemical parameters, such as creatinine, electrolytes or aminotransferases during euthyroidism, may show significant deterioration of these parameters during hypothyroidism. The presence of other diseases should be considered before submitting these patients to LT4 withdrawal. 